\title{
Pressure Effects on Single Wall Carbon Nanotube Bundles
}

\author{
P. V. Teredesai (a), A. K. Sood (a, b), S. M. Sharma (c), S. Karmakar (c), \\ S. K. Sikka (c), A. Govindaraj (b), and C. N. R. Rao (b)
}

(a) Department of Physics, Indian Institute of Science, Bangalore-560 012, India

(b) Chemistry and Physics of Materials Unit, Jawaharlal Nehru Center

for Advanced Scientific Research, Jakkur Campus, Jakkur P.O., Bangalore-560 064, India

(c) High Pressure Physics Division, Bhabha Atomic Research Center, Mumbai-400 085, India

\begin{abstract}
We report high pressure Raman studies on single wall carbon nanotube bundles under hydrostatic conditions using two different pressure transmitting media, alcohol mixture and pure water. The radial and tangential modes show a blue shift when SWNT bundle is immersed in the liquids at ambient pressures. The pressure dependence of the radial modes is the same in both liquids. However, the pressure derivatives $\mathrm{d} \omega / \mathrm{d} P$ of the tangential modes are slightly higher for the water medium. Raman results are compared with studies under non-hydrostatic conditions and with recent high-pressure X-ray studies. It is seen that the mode frequencies of the recovered sample after pressure cycling from $26 \mathrm{GPa}$ are downshifted by $\sim-10 \mathrm{~cm}^{-1}$ as compared to the starting sample.
\end{abstract}

Introduction Currently single wall carbon nanotubes (SWNT) are receiving focussed attention as they are ideal candidates for future nanoscale electronic and mechanical devices due to their unique structural and electronic properties [1]. SWNT are unusually tough materials bearing enormous flexibility in terms of complete structural reversibility [2, 3]. Other remarkable properties include one-dimensional conduction, tunability between semiconducting and metallic states [1], electric field induced electron emission [4-6] and unique capillary behavior [7, 8]. The electronic properties of SWNT can be controlled by the structure of nanotubes, by various deformations of their geometries and also by intertube interactions. The vibrational properties get influenced by the intertube interactions between the nanotubes arranged on a two-dimensional triangular lattice [9]. These interactions can be tuned by applying external pressures. In addition, high-pressure experiments provide information about structural stability and pressure-induced phase transitions. Raman spectroscopy is a powerful probe to study the pressure effects getting reflected in the vibrational spectra of the SWNT. There are two prominent features in the Raman spectra of nanotubes: one at low frequencies near $\sim 170 \mathrm{~cm}^{-1}$ associated with the symmetric radial breathing mode of the tubes and the other near $\sim 1590 \mathrm{~cm}^{-1}$ corresponding to the tangential $\mathrm{C}-\mathrm{C}$ stretching vibration. The radial mode frequency depends sensitively on the diameter of the tubes and intertube interactions [10].

The elastic properties of nanotubes are highly anisotropic $[11,12]$. The tube is extremely rigid along the tube axis as expected because any distortion along the axis is equivalent to the in-plane distortion of graphite. The elastic modulus along the radial 
direction is almost three times smaller than that along the tube axis [13]. The tubes can thus be easily distorted perpendicular to their axis and can result in the contact area being flattened on bringing two nanotubes closer [14] and for the observed collapsed structure at some places in the nanotube bundle [15]. High-pressure Raman studies have been reported on nanotubes by a few groups [11, 13, 16-19]. In all these studies, it was observed that the radial mode intensity diminishes significantly with pressure and the mode cannot be followed beyond $\sim 2.5 \mathrm{GPa}$, possibly due to facetting of the nanotubes. The pressure studies up to the highest pressure of $25.9 \mathrm{GPa}$ were carried out by us $[17,18]$ which brought out two more important results: (i) There is a softening of the tangential modes at $\sim 10 \mathrm{GPa}$. (ii) The effects of pressure on the Raman modes were reversible, which showed the remarkable mechanical resilience of the nanotubes up to $26 \mathrm{GPa}$. In all the studies so far, the pressure-transmitting medium was a methanolethanol mixture. An interesting result has been the comparison [16-18] of the pressure dependence of the radial mode frequency with the theoretical calculations [16] based on generalized tight-binding molecular dynamics. The results match very well with a model which assumes that the liquid does not penetrate in-between the nanotubes in a bundle. In order to see the role played by the liquid medium we have done high-pressure Raman experiments using water as a pressure-transmitting medium. In this paper, we compare these results with our earlier results obtained using alcohol mixture as a medium and recent results under non-hydrostatic conditions (no medium). We also discuss our results in the light of our recent high-pressure X-ray studies [20].

Experimental Details SWNT bundles were prepared by electric arc discharge method [21] as explained in our earlier paper [18]. Raman measurements were done using a laser line of $5145 \AA$ from an argon ion laser with a power of $\sim 25 \mathrm{~mW}$ on the sample. The scattered light collected in backscattered geometry was analyzed by a double grating spectrometer (SPEX Ramalog 5) and detected by RCA 31034 photomultiplier tube. The spectral resolution was $5 \mathrm{~cm}^{-1}$ and each data point was averaged for $5 \mathrm{~s}$ to improve the signal to noise ratio. High-pressure experiments were done using Mao-Bell type diamond anvil cell (DAC). The well-known ruby fluorescence technique [22] was used for pressure calibration. For hydrostatic conditions, alcohol mixture (16:3:1 $=$ methanol : ethanol : water) as well as high purity water were used as pressure transmitting media. Experiments have also been done without any liquid medium which corresponds to non-hydrostatic pressure conditions. In-situ high-pressure angle dispersive X-ray diffraction experiments were performed up to a pressure of $13 \mathrm{GPa}$ at the beamline BL10XU of Spring 8, using a monochromatized X-ray beam of $1 \AA$ wavelength.

\section{Results and Discussion}

Characterization of average tube diameter Figure 1 shows the X-ray diffraction pattern recorded at ambient pressure and room temperature using $\mathrm{CuK}_{\alpha}$ line $(\lambda=1.54 \AA)$. The low angle diffraction peak at $\theta=2.8^{\circ}$ corresponds to the Bragg reflection from (10) planes of the two-dimensional triangular lattice. This gives the lattice constant $a$ of the SWNT bundles of $15.65 \AA$ corresponding to the center to center distance between the nanotubes. The continuum theory of elasticity proposed by Tersoff and coworkers $[23,24]$ shows that the intertubular gap is $3.12 \AA$ at normal pressure compared to $3.35 \AA$ for the (002) graphite spacing. This implies that the average tube diameter in our nanotube bundles is $12.53 \AA$. It is known that the diameter of the nanotubes is 


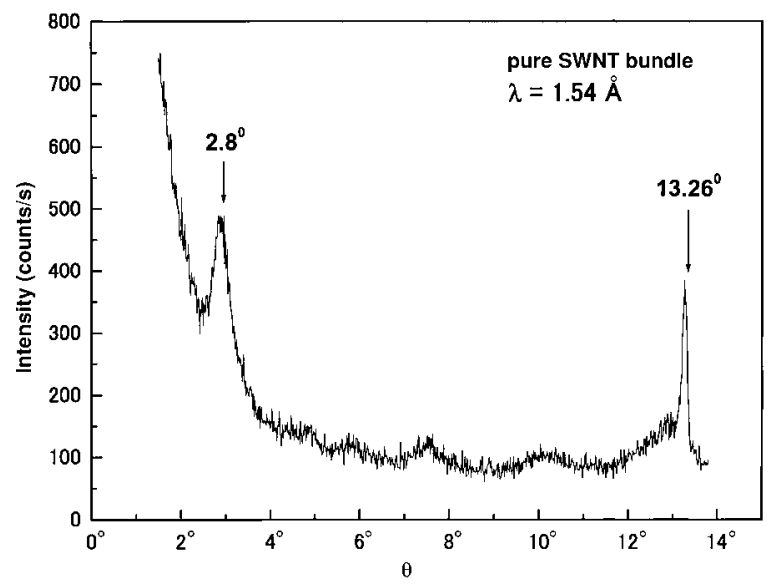

Fig 1. X-ray diffraction pattern of SWNT bundle recorded at ambient pressure and room temperature using $\mathrm{CuK}_{\alpha}$ line

related to $(n, m)$ of the tube as $d=r_{0} \sqrt{3} \sqrt{m^{2}+n^{2}+m n} / \pi$, where $r_{0}=1.42 \AA$ is the $\mathrm{C}-\mathrm{C}$ bond length of graphene sheet, $n$ and $m$ are integers defining the chiral vector, $\mathbf{C}_{\mathrm{h}}=n \mathbf{a}_{1}+m \mathbf{a}_{2}$, and $\mathbf{a}_{1}$ and $\mathbf{a}_{2}$ are the primitive vectors of the triangular lattice. Therefore, the tube diameter of $12.53 \AA$ can correspond to an achiral-armchair tube $(9,9)$ or a zigzag tube $(16,0)$ or some other combination of $n$ and $m$.

Effect of pressure transmitting media on SWNT bundles In order to understand the influence of different liquid media on SWNT bundles, we used methanol + ethanol + water $(16: 3: 1)$ and pure water for our experiments as pressure transmitting media. Figure 2 shows the Raman spectra of pure SWNT in air and inside the DAC in presence of liquid medium (alcohol mixture in Fig. 2a and water in Fig. 2b). The pressures are less than $0.1 \mathrm{GPa}$ and hence the observed shifts of the modes can be mainly attributed to the effects of the liquid rather than the external pressure applied by the anvils. The low-frequency band is best fitted to a sum of four Lorentzians with an appropriate base line and the tangential mode is fitted to a sum of five Lorentzians. The data are shown by solid circles and individual Lorentzians by dotted lines. The sum of the Lorentzians is shown by the solid lines. For the SWNT bundle in air (Fig. 2a), the radial mode frequencies are 153.7, 168.3, 175.9 and $183.2 \mathrm{~cm}^{-1}$, suggesting that tubes of different diameters are present in the sample. The observed tangential modes correspond to different symmetries as assigned by earlier polarization studies [25, 26]: $1524.8 \mathrm{~cm}^{-1}\left(\mathrm{E}_{1 \mathrm{~g}}\right)$, $1555.3 \mathrm{~cm}^{-1}\left(\mathrm{E}_{2 \mathrm{~g}}\right), 1565.7 \mathrm{~cm}^{-1}\left(\mathrm{E}_{2 \mathrm{~g}}\right), 1591.8 \mathrm{~cm}^{-1}\left(\mathrm{~A}_{1 \mathrm{~g}}+\mathrm{E}_{1 \mathrm{~g}}\right)$ and $1607.1 \mathrm{~cm}^{-1}\left(\mathrm{E}_{2 \mathrm{~g}}\right)$. Interestingly, we can see that both the radial as well as tangential modes are shifted to higher frequencies. The shift is $\sim 3 \mathrm{~cm}^{-1}$ for the radial mode and $\sim 2 \mathrm{~cm}^{-1}$ for the tangential mode at $1592 \mathrm{~cm}^{-1}$ when SWNT is immersed in alcohol. For SWNTs in water these shifts are higher; $\sim 9 \mathrm{~cm}^{-1}$ for the radial mode and $4 \mathrm{~cm}^{-1}$ for the strongest tangential mode. These shifts corroborate the recent finding [27] that the $\mathrm{D}^{*}$ band (overtone of disorder induced D band) at $\sim 2610 \mathrm{~cm}^{-1}$ in SWNT bundles shifts to higher frequencies when the SWNT bundle is immersed in liquids. The frequency shift is understood to be arising from compressive forces imposed by the liquids on the nanotubes, suggesting the potential of nanotubes as molecular sensors. This frequency shift can also arise due to charge transfer between the liquid and the nanotubes. The mechanism for the shift needs to be understood better. This is important because the mo- 


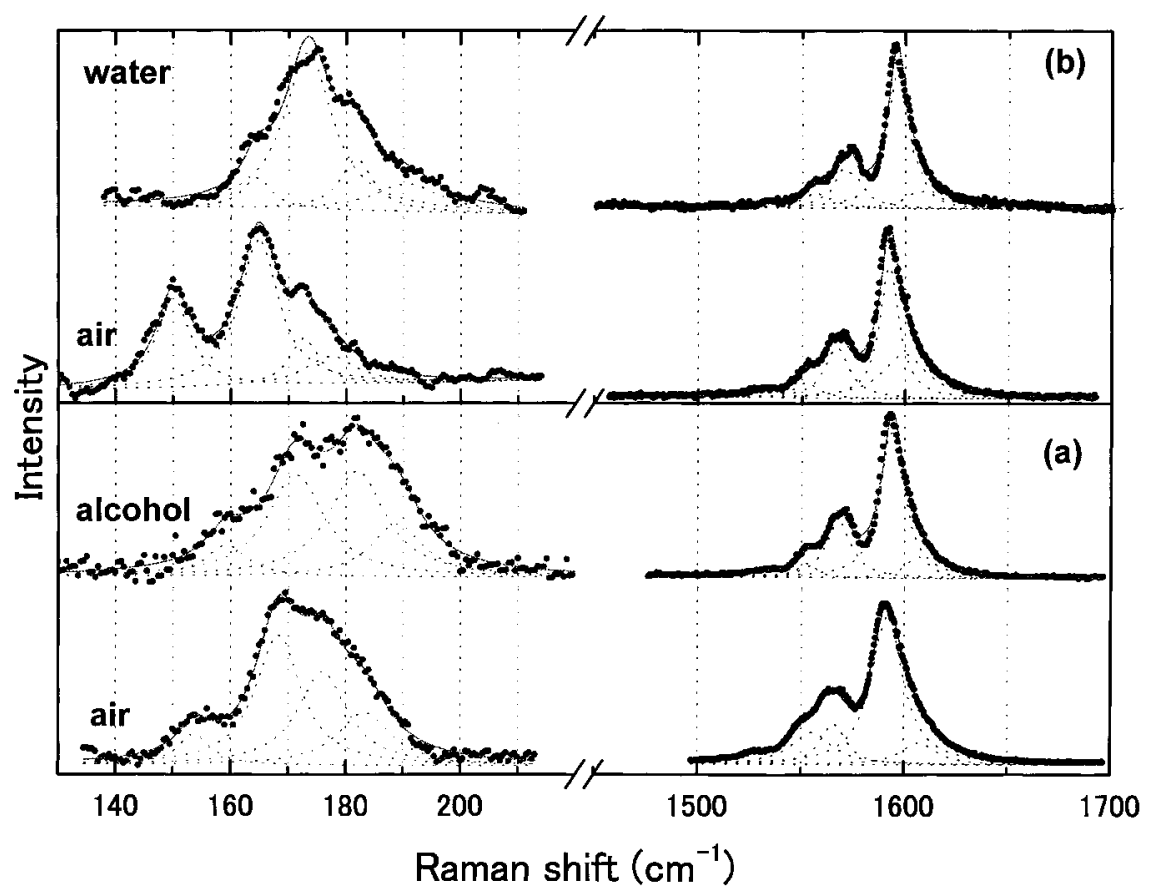

Fig. 2. a) Raman spectra of pure SWNT in air and alcohol inside DAC ( $P \approx 0.1 \mathrm{GPa})$. b) Raman spectra of pure SWNT in air and in water inside DAC $(P \approx 0 \mathrm{GPa})$. The solid circles show the data which are fitted with Lorentzians (dotted lines) and the solid lines represent their sum. Note that the batches of the samples used for alcohol and water experiments are different

lecular dynamic calculations [16] for the pressure shift of the radial mode agree remarkably well in a theoretical model, which does not include penetration of the liquid inside the bundle.

We next compare the pressure dependence of the Raman modes in two different pressure transmitting media. Figure 3 shows the two most prominent peaks observed in the radial band as a function of pressure in both increasing (solid symbols) and decreas-

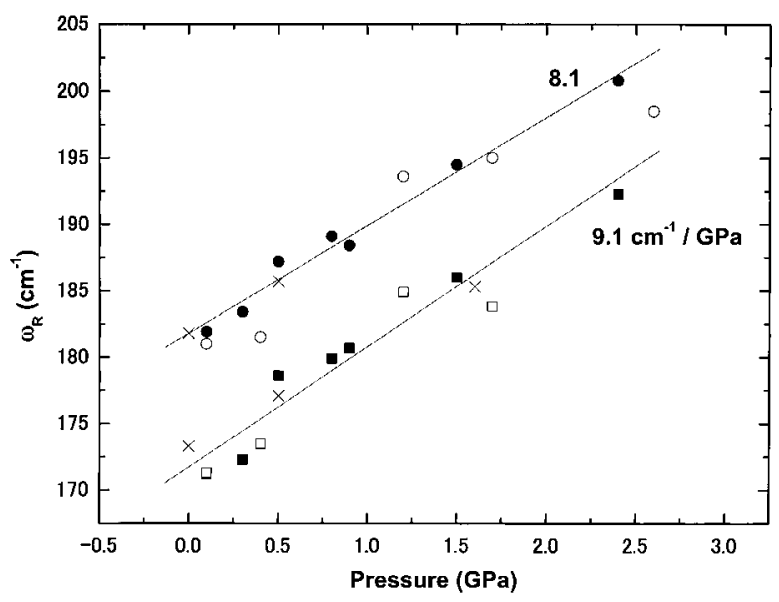

Fig. 3. Variation of two prominent peaks in the radial band with respect to pressure. The filled symbols are for increasing pressure and the open ones correspond to decreasing pressure runs. The cross marks are for the data obtained using water as pressure transmitter. The straight lines are the fits with the pressure derivatives mentioned alongside 
ing (open symbols) cycles using alcohol as pressure transmitter. The straight lines are the linear fits with pressure derivatives $\mathrm{d} \omega / \mathrm{d} P$ mentioned in the figure. The cross marks indicate the data obtained with water as pressure transmitter. The pressure derivatives $\mathrm{d} \omega / \mathrm{d} P$ for the radial band for both the transmitting media are almost the same $\left(\sim 8-9 \mathrm{~cm}^{-1} / \mathrm{GPa}\right)$ which are similar to other reports $[16,19,28]$. Beyond $2.5 \mathrm{GPa}$, radial modes could not be observed.

Next, we compare the variation of the tangential mode frequencies with pressure using alcohol and water (up to $6 \mathrm{GPa}$ ) as pressure transmitting media, in Fig. 4a for alcohol mixture [17] and in Fig. 4b for water. The solid symbols are for increasing pressure and open ones correspond to decreasing pressure runs. The lines are fits to linear equations and the fitted values of pressure derivatives $\mathrm{d} \omega / \mathrm{d} P$ are given in the figure. Three observations are noteworthy: (i) the pressure derivatives are slightly higher in water as compared to those in alcohol, (ii) there is no slope change for any of the tangential modes at $1.7 \mathrm{GPa}$. This is in contrast to the recent results of Peters et al. [19] who inferred a structural phase transition at $1.7 \mathrm{GPa}$ from the abrupt decrease in the rate of change of Raman shift with pressure for the tangential modes and disappearance of the radial mode, (iii) from Fig. $4 \mathrm{a}$, the normalized pressure derivatives defined by $\beta=\left(1 / \omega_{0}\right) \mathrm{d} \omega / \mathrm{d} P$ in units of $10^{-3} \mathrm{GPa}^{-1}$ are $3.5,3.4,3.7,3.8,3.7$, where $\omega_{0}$ is the measured frequency of the mode at zero pressure with sample immersed in alcohol. The corresponding values of $\beta$ for the SWNT with water as pressure transmitter are 2.4, $2.9,3.9,4.4$, and 5.0. It can be seen that these values are much smaller than the calculated ones [29]. Further, the calculations show that $\beta$ is different for circumferential $\left(\mathrm{A}_{1 \mathrm{~g}}, \mathrm{E}_{2 \mathrm{~g}}\right)$ and axial $\left(\mathrm{E}_{1 \mathrm{~g}}\right)$ tangential modes: 7.2 for $\mathrm{A}_{1 \mathrm{~g}}$ mode, 6.7 for $\mathrm{E}_{2 \mathrm{~g}}$ and 5.4 for $\mathrm{E}_{1 \mathrm{~g}}$ mode in units of $10^{-3} \mathrm{GPa}^{-1}$.

In our experiments carried out up to $26 \mathrm{GPa}$ we monitored the tangential mode and observed the reversibility of the Raman spectra in terms of peak positions, linewidths and intensity inside the DAC [18]. Another interesting observation is the softening of

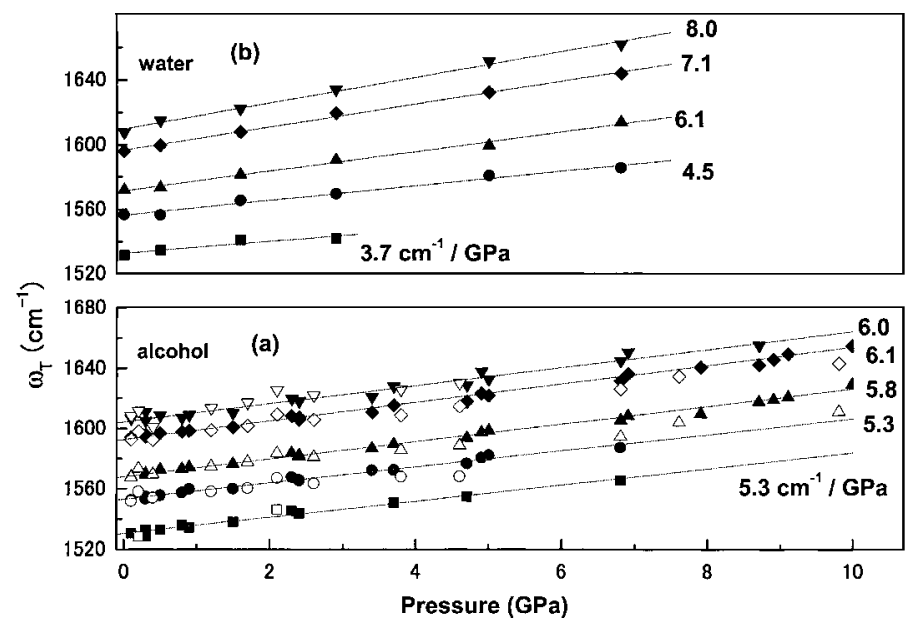

Fig. 4. Variation of tangential mode frequencies with respect to pressure, using a) alcohol and b) water as pressure transmitters. The filled symbols are for increasing pressure and open ones for decreasing pressure runs. The straight lines are linear fits and the pressure derivatives are given in the figure 


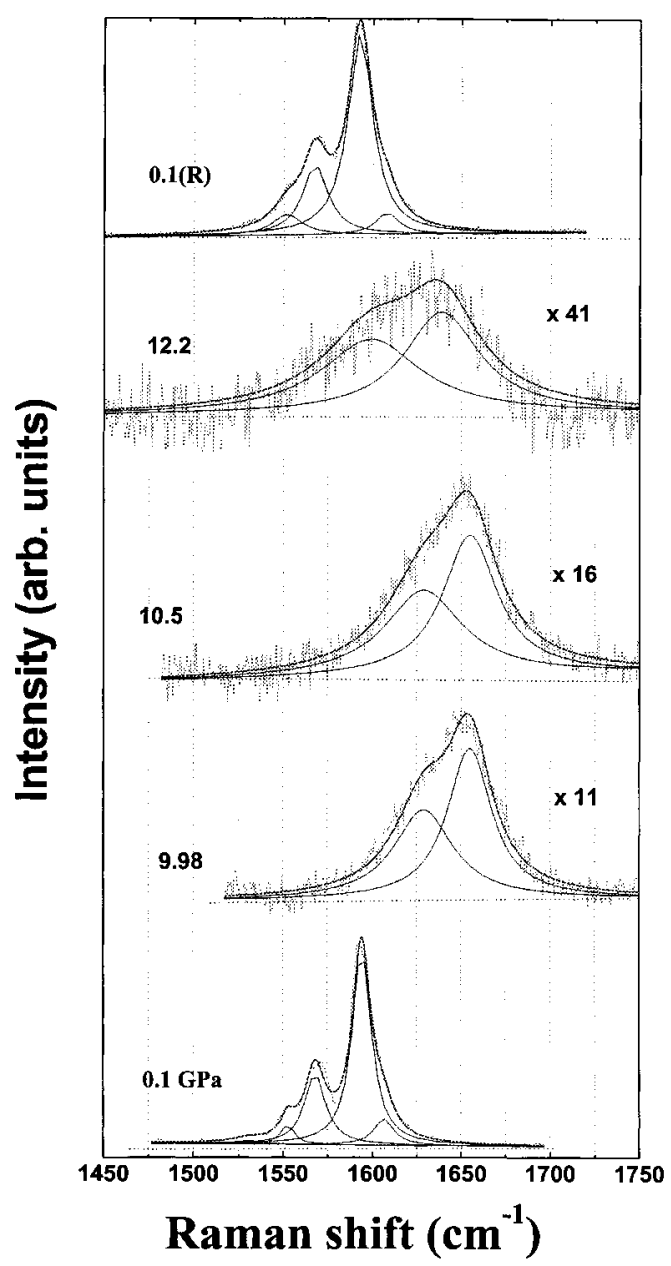

Fig. 5. Softening of the tangential mode in the hydrostatic pressure run. The data are shown alongwith the total Lorentzians (thick lines) and individual Lorentzians (dotted lines). Note that the spectra at $12.2 \mathrm{GPa}$ are red shifted with respect to the spectra at $10.5 \mathrm{GPa}$. $\mathrm{R}$ refers to the return pressure run

the tangential modes around $10 \mathrm{GPa}$ as clearly shown in Fig. 5. This softening is seen in decompression runs as well. One may speculate that this $10 \mathrm{GPa}$ transition can be a consequence of freezing of the alcohol mixture at $\sim 10 \mathrm{GPa}$. But this is ruled out in the light of high pressure experiments with water which did not show any phase transition up to $6 \mathrm{GPa}$ even though water solidifies at $\sim 1 \mathrm{GPa}$.

Comparison of the pure SWNT with pressure cycled SWNT Here we examine the complete reversibility of the Raman spectra in greater detail for both the radial as well as tangential modes. Interestingly, the Raman spectra in the decreasing pressure run at $\sim 0.1 \mathrm{GPa}$ inside the DAC agree well with the spec-

tra at similar pressures in the increasing pressure run. However, the pressure-cycled recovered sample outside the DAC shows a Raman spectrum (Fig. 6) which is downshifted by $\sim 6-10 \mathrm{~cm}^{-1}$ with respect to the starting sample. Also there is a small loss of intensity and broadening of the peaks. This implies that some structural relaxation/modification (e.g. polymerization) [30] has taken place in pressure runs which needs to be examined further.

SWNT under non-hydrostatic pressure We have explored the response of SWNT to non-hydrostatic pressure up to $30 \mathrm{GPa}$. Figure 7 shows the Raman spectra for the tangential modes at various pressures. Surprisingly, here too we find complete reversibility of SWNT bundles on decompression. This is in contrast to the observation by Chesnokov et al. [14] that there is irreversible transformation of SWNT to graphite under nonhydrostatic condition at $\sim 10 \mathrm{GPa}$. The pressure derivatives of the tangential mode frequencies are almost a factor of two larger as compared to the hydrostatic case. A detailed analysis will be published later [31]. 


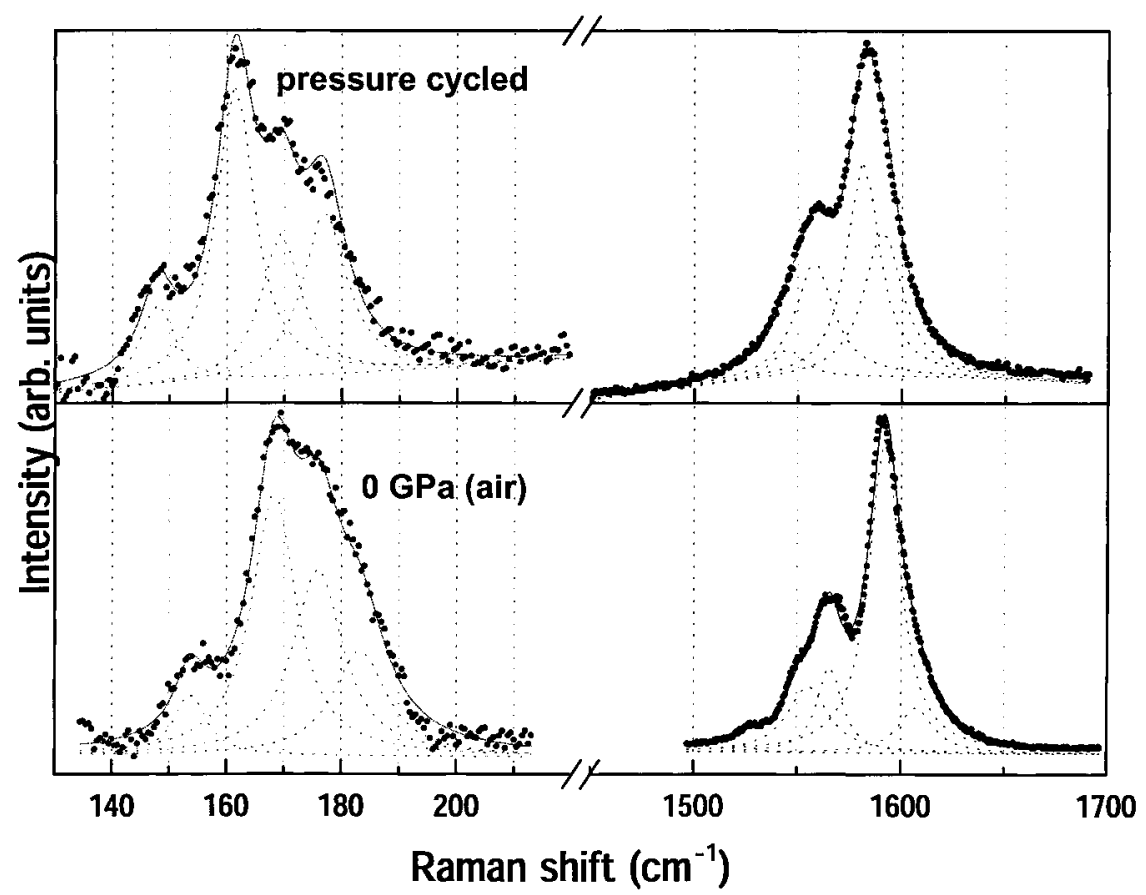

Fig. 6. Raman spectra of the pure SWNT and pressure-cycled recovered sample from 26 GPa under hydrostatic conditions. The data are shown by filled circles with the total Lorentzians shown by solid lines and individual Lorentzians shown by dotted lines

In-situ X-ray diffraction measurements We have carried out in-situ high-pressure X-ray measurements on SWNT bundles under hydrostatic conditions up to $13 \mathrm{GPa}$ [20]. We can see that the diffraction line corresponding to (10) plane of the two-dimensional triangular lattice of SWNT bundles disappears completely at $10 \mathrm{GPa}$ and appears on decompression. This can be associated to a reversible loss of translational coherence at $10 \mathrm{GPa}$. Also there is no increase in the diffraction peak intensity corresponding to (002) planes of graphite ruling out the possibility of graphitization. Further at $9.5 \mathrm{GPa}$, we see a sudden increase in the $d_{10}$ spacing indicating structural relaxation, corroborating the softening of tangential modes at $\sim 10 \mathrm{GPa}[18]$.

Conclusions Raman frequencies are blue shifted when SWNT bundles are immersed in alcohol mixture and water. The exact mechanism of this shift in terms of internal pressure exerted by the liquid vis-a-vis the charge transfer between the liquid and the nanotubes needs to be understood. The pressure derivatives of the tangential modes in water as pressure transmitter are slightly higher than the corresponding values in alcohol. The pressure-cycled sample from $26 \mathrm{GPa}$ outside the diamond anvil cell shows Raman modes which are downshifted as compared to the starting sample, implying structural relaxation. The reversibility of pressure effects is observed under non-hydrostatic pressure conditions as well. Our recent high-pressure X-ray studies show that the translational coherence in the bundle is lost around $10 \mathrm{GPa}$, which is reversible on decompression. It will be very interesting to perform high-pressure ex- 


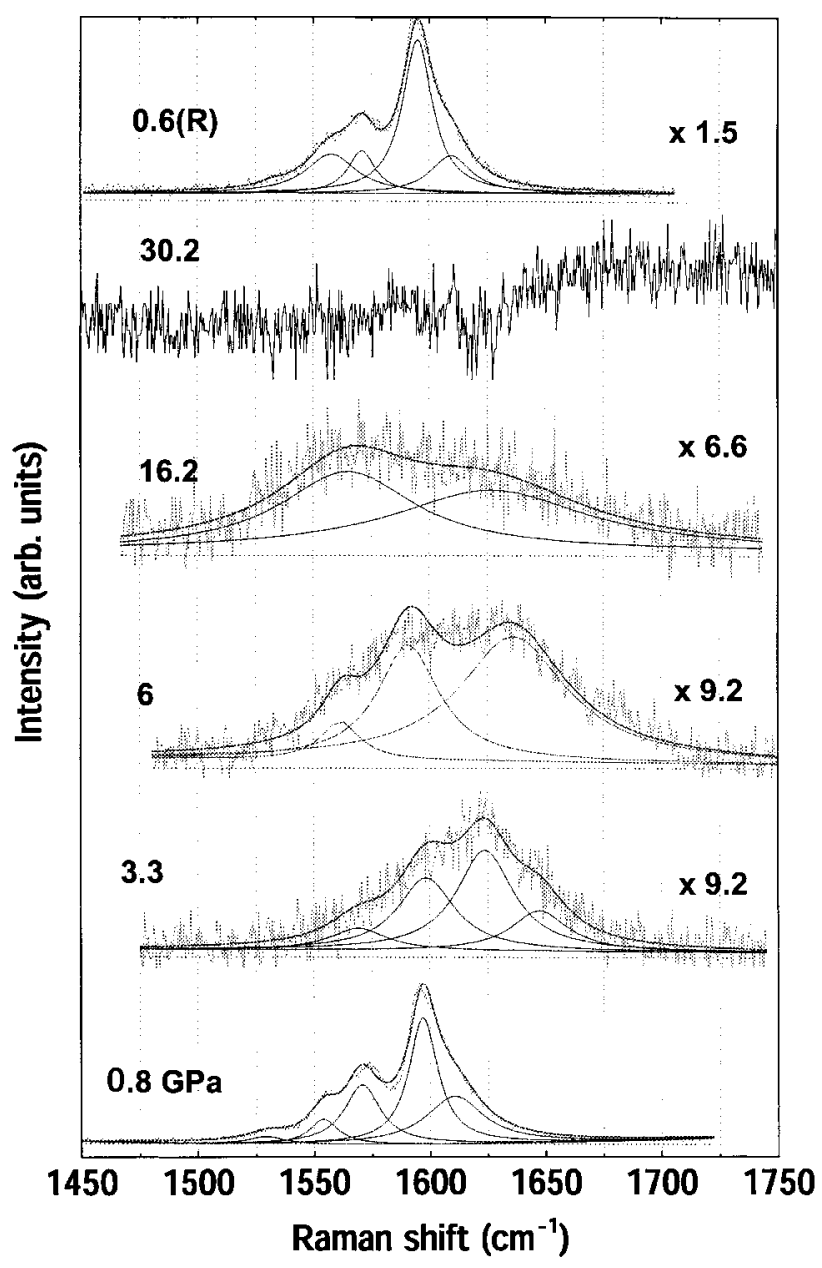

Fig. 7. Raman spectra of the tangential modes of SWNT at various pressures under nonhydrostatic conditions. The data are shown alongwith the total Lorentzian (thick lines) and individual lines (dotted lines). $\mathrm{R}$ refers to the return pressure run

periments using helium as pressure transmitting medium. We hope that our experiments will inspire to carry out molecular dynamic simulations of SWNT bundles up to high pressures of $30 \mathrm{GPa}$.

Acknowledgements A. K. S. thanks the Department of Science and Technology, India and P. V. T. thanks the Council for Scientific and Industrial Research, India for financial assistance.

\section{References}

[1] M. S. Dresselhaus, G. Dresselhaus, and P. C. EkLund, Science of Fullerenes and Carbon Nanotubes, Chap. 19, Academic Press, New York 1996.

[2] S. Ijima, C. Barbec, A. Maiti, and J. Bernholc, J. Chem. Phys. 104, 2089 (1996).

[3] B. I. Yakobson, C. J. Barbec, and J. Bernholc, Phys. Rev. Lett. 76, 2511 (1996).

[4] S. J. Tans, A. R. M. Verschueren, and C. DekKer, Nature 393, 49 (1998).

[5] S. Fan, M. G. Chapline, N. R. Franklin, T. W. Tombler, A. M. Cassell, and H. Dai, Science 283, 512 (1999). 
[6] J. M. Kiм, Bull. Amer. Soc. 45, 344 (2000).

[7] M. R. Pederson and J. Q. Broughton, Phys. Rev. Lett. 69, 2689 (1992).

[8] S. IJIMA and T. ICHIHASHI, Nature (London) 363, 603 (1993).

[9] A. Thess, R. Lee, P. Nikolaev, H. Dai, P. Petit, J. Robert, C. Xu, Y. H. Lee, S. G. Kim, A. G. Rinzler, D. T. Colbert, G. E. Scuseria, D. Tomanek, J. E. Fischer, and R. E. Smalley, Science 273, 483 (1996).

[10] S. BANDOw and S. AsaKa, Phys. Rev. Lett. 80, 3779 (1998).

[11] C. Thomsen, S. Reich, A. R. Goñi, H. Jantoljak, P. M. Rafailov, I. Loa, K. Syassen, A. Journet, and P. BERNIER, phys. stat. sol. (b) 215, 435 (1999).

[12] J. P. Lu, Phys. Rev. Lett. 79, 1297 (1997).

[13] S. Reich, H. Jantolijak, and C. Thomsen, Phys. Rev. B 61, R13389 (2000).

[14] S. A. Chesnokov, V. A. Nalimova, A. G. Rinzler, R. E. Smalley, and J. E. Fischer, Phys. Rev. Lett. 82, 343 (1999).

[15] Y. Q. Zhu, T. Sekine, T. Kobayashi, E. Takazawa, M. Terrones, and H. Terrones, Chem. Phys. Lett. 287, 689 (1998).

[16] U. D. Venkateswaran, A. M. Rao, E. Richter, M. Menon, A. Rinzler, R. E. Smalley, and P. C. EKLUND, Phys. Rev. B 59, 10928 (1999).

[17] A. K. Sood, P. V. Teredesai, D. V. S. Muthu, R. Sen, A. Govindaraj, and C. N. R. Rao, phys. stat. sol. (b) 215, 393 (1999).

[18] P. V. Teredesai, A. K. Sood, D. V. S. Muthu, R. Sen, A. Govindaraj, and C. N. R. Rao, Chem. Phys. Lett. 319, 296 (2000).

[19] M. J. Peters, L. E. McNeil, J. P. Lu, and D. Kahn, Phys. Rev. B 61, 5989 (2000).

[20] S. M. Sharma, S. Karmakar, S. K. Sikka, P. V. Teredesai, A. K. Sood, A. Govindaraj, and C. N. R. RaO, to be published.

[21] C. Journet, W. K. Maser, P. Bernier, A. Loiseau, M. Lamy de la Chapelle, S. Lefrant, P. Denierd, R. LEE, and J. E. FISCHER, Nature (London) 388, 756 (1997).

[22] R. A. Forman, G. J. Piermarini, J. D. Barnet, and S. Block, Science 176, 84 (1972).

[23] J. Tersoff, Phys. Rev. B 46, 15546 (1992).

[24] J. Tersoff and R. S. Ruoff, Phys. Rev. Lett. 73, 676 (1994).

[25] A. M. Rao, E. Richter, S. Bandow, B. Chase, P. C. Eklund, K. A. Williams, S. Fang, R. Subbaswamy, M. Menon, A. Thess, R. E. Smalley, G. Dresselhaus, and M. S. Dresselhaus, Science 275, 187 (1997).

[26] A. Jorio, G. Dresselhaus, M. S. Dresselhaus, M. Souza, M. S. S. Dantas, M. A. Pimenta, A. M. Rao, R. Saito, C. Liu, and H. M. Cheng, Phys. Rev. Lett. 85, 2617 (2000).

[27] J. R. Wood, M. D. Forgley, E. R. Meurs, A. D. Prins, T. Peiss, D. J. Dunstan, and H. D. Wagner, J. Phys. Chem. B 103, 10388 (1999).

[28] U. D. Venkateswaran, E. A. Bransden, U. Schlecht, A. M. Rao, E. Richter, I. Loa, K. SyasSEN, and P. C. EKLund, Proc. 9th Internat. Conf. High Pressure Semiconductor Physics, Sapporo (Japan), September 24-28, 2000; phys. stat. sol. (b) 223, 225 (2001).

[29] D. KAHN and J. P. Lu, Phys. Rev. B 60, 6535 (1999).

[30] T. Yildirim, O. Gulseren, C. K. Kilic, and S. Ciraci, Phys. Rev. B 62, 12648 (2000).

[31] P. V. Teredesai and A. SoOD, to be published. 
